\title{
A PRIORI ERROR ESTIMATES FOR MIXED FINITE ELEMENT APPROXIMATIONS OF THE ACOUSTIC WAVE EQUATION*
}

\author{
ELEANOR W. JENKINS ${ }^{\dagger}$, BÉATRICE RIVIÈRE $^{\dagger}$, AND MARY F. WHEELER ${ }^{\dagger}$
}

\begin{abstract}
In this paper we derive optimal a priori $L^{\infty}\left(L^{2}\right)$ error estimates for mixed finite element displacement formulations of the acoustic wave equation. The computational complexity of this approach is equivalent to the traditional mixed finite element formulations of the second order hyperbolic equations in which the primary unknowns are pressure and the gradient of pressure. However, the displacement formulations with the physical variables of interest, displacement and pressure, requires less regularity on the displacement.
\end{abstract}

Key words. acoustic wave equation, mixed finite elements, error estimation

AMS subject classifications. 35L05, 65M12, 65M60, 65M15

PII. S0036142901388068

1. Introduction. There is significant interest in simulating the effects of wave propagation in heterogeneous media to aid in the interpretation of field data and to predict the damage patterns due to earthquakes. Simulated waveform data (seismograms) computed for an assumed earth model are compared against the recorded data. If the match is unacceptable, the model is perturbed, and the simulation is redone and compared again. This procedure is implemented formally by global optimization techniques [19] resulting in a description of an earth model (with its associated uncertainties) that explains the observations. Thus there is a need for a fast and accurate simulation technique that can be used for real time analysis of seismograms.

In the past, wave simulation has been successfully modeled using finite difference methods $[10,13]$, but these solutions have been expensive to compute. The use of structured finite differences in simulating earthquake responses in the Los Angeles Basin requires 35 billion grid points [3], which emphasizes the need for unstructured meshes. The staggered grid approach described in [10] may be used to solve problems on the order of millions using a workstation, but memory optimization routines must be used and it is unclear that the method is easily parallelized.

Finite element discretization methods have the advantage of handling complex geometries and straightforward local discretization techniques using error indicators. It is also easy to incorporate free surface boundary conditions and nonmatching grids. In [13], Marfurt concludes that finite element methods may be the most cost-effective way to simulate wave fields.

Previous attempts at wave simulation using finite elements have used continuous Galerkin methods [2, 3, 9, 13, 18], discontinuous Galerkin methods [11, 17], and mixed finite element methods $[7,8]$. We propose mixed finite element displacement formulations for solving the acoustic wave equation, which are described below. These approximations can be defined for general meshes.

\footnotetext{
*Received by the editors April 18, 2001; accepted for publication (in revised form) April 25, 2002; published electronically November 14, 2002. This research was supported by the NSF through NPACI and the National Partnership for Advanced Computational Infrastructure, grant 10152711. http://www.siam.org/journals/sinum/40-5/38806.html

${ }^{\dagger}$ Center for Subsurface Modeling, Texas Institute for Computational and Applied Mathematics, University of Texas at Austin, ACES 5.340, 201 E. 24th Street, Austin, TX 78712 (lea@ticam.utexas.edu, riviere@ticam.utexas.edu,mfw@ticam.utexas.edu).
} 
Let $\Omega$ be a bounded domain in $\mathbb{R}^{n}, n=1,2,3$, with boundary $\partial \Omega=\Gamma_{D} \cup \Gamma_{N}$. The general form of the wave equation is

$$
\begin{aligned}
\rho \boldsymbol{u}_{t t}-\nabla \cdot \tilde{\boldsymbol{\tau}} & =\boldsymbol{f} & & \text { in } \Omega \times(0, T), \\
\nabla \cdot \boldsymbol{u} & =0 & & \text { on } \Gamma_{D} \times(0, T), \\
\boldsymbol{u} \cdot \boldsymbol{\nu} & =0 & & \text { on } \Gamma_{N} \times(0, T), \\
\boldsymbol{u}(\cdot, 0) & =\boldsymbol{u}_{0} & & \text { in } \Omega, \\
\boldsymbol{u}_{t}(\cdot, 0) & =\boldsymbol{u}_{1} & & \text { in } \Omega,
\end{aligned}
$$

where $\boldsymbol{u}$ is the displacement, $\rho$ is the density, and $\tilde{\boldsymbol{\tau}}$ is the stress tensor given by the generalized Hooke's law $\tilde{\boldsymbol{\tau}}=\lambda(\nabla \cdot \boldsymbol{u}) \tilde{\boldsymbol{I}}+\mu\left(\nabla \boldsymbol{u}+(\nabla \boldsymbol{u})^{T}\right)$. Here $\lambda>0$ and $\mu$ are the Lamé coefficients characterizing the material. We let $\boldsymbol{f}$ represent a general source term, and let $\boldsymbol{u}_{0}$ and $\boldsymbol{u}_{1}$ be the initial conditions on displacements and velocities, and we assume that $\boldsymbol{f}, \boldsymbol{u}_{0}$, and $\boldsymbol{u}_{1}$ are smooth enough so that there is a unique solution $\boldsymbol{u} \in \mathcal{C}^{2}((0, T) \times \Omega)$ to $(1.1)-(1.5)[12]$.

The acoustic problem is the limiting case with $\mu=0$. In this case, (1.1) becomes

$$
\rho \boldsymbol{u}_{t t}-\nabla \cdot(\lambda(\nabla \cdot \boldsymbol{u}) \tilde{\boldsymbol{I}})=\boldsymbol{f} .
$$

We assume that $\rho$ and $\lambda$ are bound above and below by the positive constants $\rho_{0}, \rho_{1}$, $\lambda_{0}$, and $\lambda_{1}$, respectively.

A standard approach in geophysics modeling is to solve a scalar wave equation. Here, since $p=\lambda \nabla \cdot \boldsymbol{u}$, we have $p_{t t}=\lambda \nabla \cdot \boldsymbol{u}_{t t}$. We substitute this expression into (1.6) to obtain

$$
p_{t t}-\lambda \nabla \cdot \frac{1}{\rho}(\nabla p)=\tilde{f}
$$

where $\tilde{f}=\nabla \cdot \boldsymbol{f}$.

A priori error estimates for solving (1.7) with a constant $\lambda$ were obtained by Cowsar, Dupont, and Wheeler $[7,8]$. We propose an alternative mixed finite elements displacement formulation that requires less regularity on the displacement solution than the approach in $[7,8]$. We describe this method in section 3 . We derive the error estimates for the continuous-in-time problem in section 4. For the discrete-in-time problem, stability results and error estimates are obtained in section 5 . In section 6 we present conclusions.

2. Preliminaries. In this section we describe the notation used in this paper, we introduce the functional spaces, two projection operators and their approximation properties, and we recall Gronwall's inequality, which is needed in the error analysis.

2.1. Inner products and norms. The $L^{2}$ inner product over $\Omega$ is defined as

$$
(u, v)=\int_{\Omega} u v d \Omega
$$

and we denote by $\|\cdot\|_{L^{2}}$ the $L^{2}$ norm over $\Omega$, i.e., $\|u\|_{L^{2}(\Omega)}=(u, u)^{\frac{1}{2}}$. The inner product over the boundary $\partial \Omega$ is denoted

$$
\langle u, v\rangle=\int_{\partial \Omega} u v d \Omega
$$


for $u, v \in H^{\frac{1}{2}+\epsilon}(\Omega)$, with $\epsilon>0$. The time-space norm $\|\cdot\|_{L^{2}\left(0, T ; L^{2}(\Omega)\right)}$ is defined as

$$
\|u\|_{L^{2}\left(0, T ; L^{2}(\Omega)\right)}=\|u\|_{L^{2}\left(L^{2}\right)}=\left(\int_{0}^{T}\|u\|_{L^{2}(\Omega)}^{2}\right)^{\frac{1}{2}} .
$$

The time-space norm $\|\cdot\|_{L^{\infty}\left(L^{2}\right)}$ is similarly defined.

2.2. Gronwall's inequality. We recall Gronwall's inequality, which states that if $y \geq 0$ satisfies $y_{t} \leq k y(t)+h(t)$ for $0 \leq t \leq \tau$, where $k \geq 0$ is a constant and $h(t) \geq 0, h \in L^{1}((0, \tau))$, then

$$
y(t) \leq e^{k \tau}\left[y(0)+\int_{0}^{\tau} h(s) d s\right]
$$

for all $t \in[0, \tau][14]$.

2.3. Functional spaces and projections. We define the standard Sobolev spaces for mixed methods:

$$
\begin{aligned}
\boldsymbol{H}(\Omega, \operatorname{div}) & =\left\{\boldsymbol{v}: \boldsymbol{v} \in\left(\boldsymbol{L}^{2}(\Omega)\right)^{n}, \nabla \cdot \boldsymbol{v} \in \boldsymbol{L}^{2}(\Omega)\right\}, \\
\boldsymbol{V} & =\left\{\boldsymbol{v} \in \boldsymbol{H}(\Omega, \operatorname{div}):\left.\boldsymbol{v} \cdot \boldsymbol{\nu}\right|_{\Gamma_{N}}=0\right\}, \\
W & =H^{\frac{1}{2}+\epsilon}(\Omega) \text { for any } \epsilon>0 .
\end{aligned}
$$

Let $\left\{\mathcal{E}_{h}\right\}_{h>0}$ be a quasi-uniform family of finite element partitions of $\Omega$, where $h$ is the maximal element diameter. Let $\boldsymbol{V}_{h} \times W_{h}$ be any of the usual mixed finite element approximating subspaces of $\boldsymbol{V} \times W$, that is, the Raviart-Thomas-Nedelec spaces $[15,16]$, Brezzi-Douglas-Marini spaces [5], or Brezzi-Douglas-Fortin-Marini spaces [4].

Each of these mixed spaces has a projection operator $\Pi_{h}: \boldsymbol{H}(\Omega, \operatorname{div}) \rightarrow \boldsymbol{V}_{h}$ such that for any $\boldsymbol{z} \in \boldsymbol{H}(\Omega$, div)

$$
\left(\nabla \cdot \Pi_{h} \boldsymbol{z}, w\right)=(\nabla \cdot \boldsymbol{z}, w) \quad \forall w \in W_{h} .
$$

In addition, if $\boldsymbol{z} \in \boldsymbol{H}(\Omega$, div $) \cap \boldsymbol{H}^{k}(\Omega)$, we also have

$$
\left\|\Pi_{h} \boldsymbol{z}-\boldsymbol{z}\right\|_{0} \leq C h^{j}\|\boldsymbol{z}\|_{j}, \quad 1 \leq j \leq k,
$$

where $k$ is associated with the degree of the polynomial, and $\|\cdot\|_{s}$ is the standard Sobolev norm on $\left(\boldsymbol{H}^{s}(\Omega)\right)^{n}[1]$.

Let $\mathcal{P}_{h}$ be the $L^{2}$ projection of $W$ onto $W_{h}$ such that

$$
\left(\mathcal{P}_{h} \phi, w\right)=(\phi, w) \quad \forall \phi \in W, \quad \forall w \in W_{h} .
$$

In addition, if $\phi \in W \cap H^{k}(\Omega)$, then we also have

$$
\left\|\mathcal{P}_{h} \phi-\phi\right\|_{s} \leq C h^{j-s}\|\phi\|_{j}, \quad 0 \leq s \leq k, \quad 0 \leq j \leq k .
$$

3. Model problem and scheme. We observe that $\nabla \cdot(\lambda(\nabla \cdot \boldsymbol{u}) \tilde{\boldsymbol{I}})=\nabla(\lambda \nabla \cdot \boldsymbol{u})$ so that (1.6) may be rewritten as

$$
\rho \boldsymbol{u}_{t t}-\nabla(\lambda \nabla \cdot \boldsymbol{u})=f .
$$


By introducing $p=\lambda \nabla \cdot \boldsymbol{u}$, we present (3.1) in a mixed finite element form:

$$
\begin{aligned}
\rho \boldsymbol{u}_{t t}-\nabla p=\boldsymbol{f} & \text { in } \Omega \times(0, T), \\
\lambda^{-1} p=\nabla \cdot \boldsymbol{u} & \text { in } \Omega \times(0, T) .
\end{aligned}
$$

A similar approach for the linear elasticity problem has been presented by Brezzi and Fortin in [6].

Let $\boldsymbol{v} \in \boldsymbol{V}$ and $w \in W$. If we multiply (3.2) and (3.3) by $\boldsymbol{v}$ and $w$, respectively, and integrate over $\Omega$ we get the weak formulation

$$
\begin{aligned}
\left(\rho \boldsymbol{u}_{t t}, \boldsymbol{v}\right)-(\nabla p, \boldsymbol{v}) & =(\boldsymbol{f}(t), \boldsymbol{v}) & & \forall \boldsymbol{v} \in \boldsymbol{V}, \\
\left(\lambda^{-1} p, w\right)-(\nabla \cdot \boldsymbol{u}, w) & =0 & & \forall w \in W .
\end{aligned}
$$

Clearly, if $\boldsymbol{u}, p$ satisfy (3.2) and (3.3), then $\boldsymbol{u}, p$ satisfy (3.4) and (3.5).

If we integrate by parts in (3.4) we get, for any $\boldsymbol{v} \in \boldsymbol{V}$,

$$
(\nabla p, \boldsymbol{v})=\langle p, \boldsymbol{v} \cdot \nu\rangle-(p, \nabla \cdot \boldsymbol{v})=-(p, \nabla \cdot \boldsymbol{v}),
$$

and the weak formulation becomes the following:

For any $t \geq 0$, find $(\boldsymbol{u}(t), p(t)) \in \boldsymbol{V} \times W$ such that

$$
\begin{aligned}
& (\boldsymbol{u}(0), \boldsymbol{v})=\left(\boldsymbol{u}_{0}, \boldsymbol{v}\right) \quad \forall \boldsymbol{v} \in \boldsymbol{V}, \\
& \left(\boldsymbol{u}_{t}(0), \boldsymbol{v}\right)=\left(\boldsymbol{u}_{1}, \boldsymbol{v}\right) \quad \forall \boldsymbol{v} \in \boldsymbol{V}, \\
& \left(\lambda^{-1} p(0), w\right)=\left(\nabla \cdot \boldsymbol{u}_{0}, w\right) \quad \forall w \in W, \\
& \left(\rho \boldsymbol{u}_{t t}(t), \boldsymbol{v}\right)+(p(t), \nabla \cdot \boldsymbol{v})=(\boldsymbol{f}(t), \boldsymbol{v}) \quad \forall \boldsymbol{v} \in \boldsymbol{V}, \quad \forall t>0, \\
& \left(\lambda^{-1} p(t), w\right)-(\nabla \cdot \boldsymbol{u}(t), w)=0 \quad \forall w \in W, \quad \forall t>0 .
\end{aligned}
$$

Note that in $[7,8]$ it is necessary that $\nabla p \in \boldsymbol{H}(\Omega$, div $)$ so that $\nabla \cdot \boldsymbol{u} \in H^{2}(\Omega)$. Here, we require only that $\nabla \cdot \boldsymbol{u} \in H^{\frac{1}{2}}(\Omega)$. It is clear that the solution $\boldsymbol{u} \in \mathcal{C}^{2}((0, T) \times \Omega)$ of problem (1.1)-(1.5) with $p=\lambda \nabla \cdot \boldsymbol{u}$ is a solution to (3.6)-(3.10). The uniqueness is provided by the following lemma.

LEMma 3.1. Let $\left(\boldsymbol{u}_{a}, p_{a}\right)$ and $\left(\boldsymbol{u}_{b}, p_{b}\right)$ be two solutions of (3.6)-(3.10). Then $\boldsymbol{u}_{a}=\boldsymbol{u}_{b}$ and $p_{a}=p_{b}$.

Proof. Let $\boldsymbol{\chi}=\boldsymbol{u}_{a}-\boldsymbol{u}_{b}$ and $\psi=p_{a}-p_{b}$. Then by subtracting the equations satisfied by these solutions we have

$$
\begin{array}{rlrl}
(\chi(0), \boldsymbol{v}) & =0 & \forall \boldsymbol{v} \in \boldsymbol{V}, & \\
\left(\chi_{t}(0), \boldsymbol{v}\right)=0 & \forall \boldsymbol{v} \in \boldsymbol{V}, & \\
\left(\lambda^{-1} \psi(0), w\right)=0 & \forall w \in W, & \\
\left(\rho \boldsymbol{\chi}_{t t}(t), \boldsymbol{v}\right)+(\psi(t), \nabla \cdot \boldsymbol{v}) & =0 & \forall \boldsymbol{v} \in \boldsymbol{V}, & \forall t>0, \\
\left(\lambda^{-1} \psi(t), w\right)-(\nabla \cdot \boldsymbol{\chi}(t), w) & =0 & \forall w \in W, & \forall t>0 .
\end{array}
$$

We differentiate (3.15) with respect to time:

$$
\left(\lambda^{-1} \psi_{t}, w\right)-\left(\nabla \cdot \chi_{t}, w\right)=0 \quad \forall w \in W, \quad \forall t>0 .
$$

We choose $\boldsymbol{v}=\chi_{t}$ and $w=p$. Thus (3.14) and (3.16) become

$$
\begin{aligned}
\left(\rho \boldsymbol{\chi}_{t t}, \boldsymbol{\chi}_{t}\right)+\left(\psi, \nabla \cdot \boldsymbol{\chi}_{t}\right) & =0 \\
\left(\lambda^{-1} \psi_{t}, \psi\right)-\left(\nabla \cdot \boldsymbol{\chi}_{t}, \psi\right) & =0 .
\end{aligned}
$$


We add (3.17) and (3.16) so that

$$
\left(\rho \boldsymbol{\chi}_{t t}, \boldsymbol{\chi}_{t}\right)+\left(\lambda^{-1} \psi_{t}, \psi\right)=0
$$

and thus

$$
\frac{1}{2} \frac{d}{d t}\left\|\rho^{\frac{1}{2}} \chi_{t}\right\|_{L^{2}(\Omega)}^{2}+\frac{1}{2} \frac{d}{d t}\left\|\lambda^{-\frac{1}{2}} \psi\right\|_{L^{2}(\Omega)}^{2}=0
$$

We integrate (3.20) with respect to time to obtain

$$
\frac{1}{2}\left\|\rho^{\frac{1}{2}} \chi_{t}\right\|_{L^{2}(\Omega)}^{2}+\frac{1}{2}\left\|\lambda^{-\frac{1}{2}} \psi\right\|_{L^{2}(\Omega)}^{2}=C_{1},
$$

where $C_{1}$ is a constant independent of time. As (3.21) holds for any $t$, it holds in particular for $t=0$. We have from the initial data that $\left\|\chi_{t}(0)\right\|_{L^{2}(\Omega)}^{2}=\|\psi(0)\|_{L^{2}(\Omega)}^{2}=$ 0 , so $C_{1}=0$. Hence

$$
\frac{1}{2}\left\|\rho^{\frac{1}{2}} \chi_{t}\right\|_{L^{2}(\Omega)}^{2}+\frac{1}{2}\left\|\lambda^{-\frac{1}{2}} \psi\right\|_{L^{2}(\Omega)}^{2}=0
$$

and therefore

$$
\frac{1}{2}\left\|\rho^{\frac{1}{2}} \chi_{t}\right\|_{L^{2}(\Omega)}^{2}=\frac{1}{2}\left\|\lambda^{-\frac{1}{2}} \psi\right\|_{L^{2}(\Omega)}^{2}=0 .
$$

However, we assume that both $\lambda$ and $\rho$ are bounded above and below away from 0 . Hence we must have that

$$
\chi_{t}=\psi=0
$$

Now we consider $\chi_{t}=0$. We integrate with respect to time and obtain

$$
\chi=C_{2},
$$

where $C_{2}$ is a constant independent of time. This holds for any $t$; in particular, it holds for $t=0$. Again, we use the initial conditions and see that $\chi(0)=0$, and hence $C_{2}=0$. Thus $\chi=0$, which concludes the proof.

The mixed finite element approximation to $(\boldsymbol{u}(t), p(t))$ for any $t \geq 0$ is given by the functions $(\boldsymbol{U}(t), P(t)) \in \boldsymbol{V}_{h} \times W_{h}$ satisfying

$$
\begin{aligned}
& (\boldsymbol{U}(0), \boldsymbol{v})=\left(\Pi_{h} \boldsymbol{u}_{0}, \boldsymbol{v}\right) \quad \forall \boldsymbol{v} \in \boldsymbol{V}_{h}, \\
& \left(\boldsymbol{U}_{t}(0), \boldsymbol{v}\right)=\left(\Pi_{h} \boldsymbol{u}_{1}, \boldsymbol{v}\right) \quad \forall \boldsymbol{v} \in \boldsymbol{V}_{h}, \\
& (P(0), w)=(p(0), w) \quad \forall w \in W_{h}, \\
& \left(\rho \boldsymbol{U}_{t t}(t), \boldsymbol{v}\right)+(P(t), \nabla \cdot \boldsymbol{v})=(\boldsymbol{f}(t), \boldsymbol{v}) \quad \forall \boldsymbol{v} \in \boldsymbol{V}_{h}, \quad \forall t>0, \\
& \left(\lambda^{-1} P(t), w\right)-(\nabla \cdot \boldsymbol{U}(t), w)=0 \quad \forall w \in W_{h}, \quad \forall t>0 .
\end{aligned}
$$

The existence and uniqueness of a solution $(\boldsymbol{U}(t), P(t))$ to (3.24)-(3.28) is shown in the following lemma.

Lemma 3.2. A solution $(\boldsymbol{U}(t), P(t))$ to (3.24)-(3.28) exists and is unique.

Proof. Because we are operating in a finite dimensional space, it suffices to show uniqueness. Uniqueness follows directly from the proof of the previous lemma. 
4. Continuous in time a priori error estimates. In this section, we prove the convergence of the scheme (3.24)-(3.28) in the $L^{\infty}\left(L^{2}\right)$ norm. We first derive an estimate for the error in the pressure and the velocity, then for the error in the displacement.

THEOREM 4.1. For $t \geq 0$, let $(\boldsymbol{u}(t), p(t))$ be the solution of the problem (3.6)(3.10). Assume that $\boldsymbol{u}_{t} \in L^{\infty}\left(\boldsymbol{L}^{2}(\Omega)\right), \boldsymbol{u}_{t t} \in L^{2}\left(\boldsymbol{H}^{k}(\Omega)\right), p \in L^{\infty}\left(L^{2}(\Omega)\right)$, and $p_{t} \in L^{2}\left(H^{k}(\Omega)\right)$. Then there exists a constant $C$ independent of $h$ such that

$$
\begin{aligned}
\left\|\rho^{\frac{1}{2}}\left(\boldsymbol{u}_{t}-\boldsymbol{U}_{t}\right)\right\|_{L^{\infty}\left(L^{2}\right)}+\| \lambda^{-\frac{1}{2}}(p-P) & \|_{L^{\infty}\left(L^{2}\right)} \\
& \leq C h^{k}\left(\left\|\boldsymbol{u}_{t t}\right\|_{L^{2}\left(H^{k}\right)}+\left\|p_{t}\right\|_{L^{2}\left(H^{k}\right)}\right),
\end{aligned}
$$

where $k$ is associated with the degree of the finite element polynomial.

Proof. For simplification, we denote $\chi=\boldsymbol{U}-\Pi_{h} \boldsymbol{u}, \xi=P-\mathcal{P}_{h} p, \boldsymbol{\eta}=\boldsymbol{u}-\Pi_{h} \boldsymbol{u}$, and $\zeta=p-\mathcal{P}_{h} p$, where $\Pi_{h}$ and $\mathcal{P}_{h}$ have been defined in section 2.3. These definitions hold throughout the paper. If we subtract $\Pi_{h} \boldsymbol{u}$ and $\mathcal{P}_{h} p$ from (3.9), (3.10), (3.27), and (3.28) we obtain

$$
\begin{array}{rlrl}
\left(\rho \boldsymbol{\chi}_{t t}, \boldsymbol{v}\right)+(\xi, \nabla \cdot \boldsymbol{v}) & =\left(\rho \boldsymbol{\eta}_{t t}, \boldsymbol{v}\right)+(\zeta, \nabla \cdot \boldsymbol{v}) & & \forall \boldsymbol{v} \in \boldsymbol{V}_{h} \\
\left(\lambda^{-1} \xi, w\right)-(\nabla \cdot \chi, w) & =\left(\lambda^{-1} \zeta, w\right)-(\nabla \cdot \boldsymbol{\eta}, w) & \forall w \in W_{h}
\end{array}
$$

Since $\boldsymbol{\chi} \in \boldsymbol{V}_{h}$, we can set $\boldsymbol{v}=\boldsymbol{\chi}_{t}$ in (4.2):

$$
\left(\rho \boldsymbol{\chi}_{t t}, \boldsymbol{\chi}_{t}\right)+\left(\xi, \nabla \cdot \boldsymbol{\chi}_{t}\right)=\left(\rho \boldsymbol{\eta}_{t t}, \boldsymbol{\chi}_{t}\right)+\left(\zeta, \nabla \cdot \boldsymbol{\chi}_{t}\right)
$$

We then differentiate (4.3) with respect to time to obtain

$$
\left(\lambda^{-1} \xi_{t}, w\right)-\left(\nabla \cdot \chi_{t}, w\right)=\left(\lambda^{-1} \zeta_{t}, w\right)-\left(\nabla \cdot \boldsymbol{\eta}_{t}, w\right) \quad \forall w \in W_{h}
$$

As $\xi \in W_{h}$, we can set $w=\xi$ in $(4.5)$, which gives

$$
\left(\lambda^{-1} \xi_{t}, \xi\right)-\left(\nabla \cdot \chi_{t}, \xi\right)=\left(\lambda^{-1} \zeta_{t}, \xi\right)-\left(\nabla \cdot \boldsymbol{\eta}_{t}, \xi\right)
$$

Adding (4.4) and (4.6) gives

$$
\begin{aligned}
\frac{1}{2} \frac{d}{d t}\left\|\rho^{\frac{1}{2}} \chi_{t}\right\|_{L^{2}(\Omega)}^{2}+\frac{1}{2} \frac{d}{d t} & \left\|\lambda^{-\frac{1}{2}} \xi\right\|_{L^{2}(\Omega)}^{2} \\
& =\left(\rho \boldsymbol{\eta}_{t t}, \chi_{t}\right)+\left(\zeta, \nabla \cdot \chi_{t}\right)+\left(\lambda^{-1} \zeta_{t}, \xi\right)-\left(\nabla \cdot \boldsymbol{\eta}_{t}, \xi\right)
\end{aligned}
$$

If we use the definitions (2.1) and (2.3) of the $\Pi_{h}$ and $\mathcal{P}_{h}$ projections, we obtain

$$
\frac{1}{2} \frac{d}{d t}\left\|\rho^{\frac{1}{2}} \chi_{t}\right\|_{L^{2}(\Omega)}^{2}+\frac{1}{2} \frac{d}{d t}\left\|\lambda^{-\frac{1}{2}} \xi\right\|_{L^{2}(\Omega)}^{2}=\left(\rho \boldsymbol{\eta}_{t t}, \chi_{t}\right)+\left(\lambda^{-1} \zeta_{t}, \xi\right) .
$$

We use the Cauchy-Schwarz inequality to bound the right-hand side of (4.8) so that

$$
\begin{aligned}
\frac{1}{2} \frac{d}{d t}\left\|\rho^{\frac{1}{2}} \chi_{t}\right\|_{L^{2}(\Omega)}^{2}+\frac{1}{2} \frac{d}{d t}\left\|\lambda^{-\frac{1}{2}} \xi\right\|_{L^{2}(\Omega)}^{2} \leq & \left\|\rho^{\frac{1}{2}} \chi_{t}\right\|_{L^{2}(\Omega)}\left\|\rho^{\frac{1}{2}} \boldsymbol{\eta}_{t t}\right\|_{L^{2}(\Omega)} \\
& +\left\|\lambda^{-\frac{1}{2}} \zeta_{t}\right\|_{L^{2}(\Omega)}\left\|\lambda^{-\frac{1}{2}} \xi\right\|_{L^{2}(\Omega)}
\end{aligned}
$$


and use the fact that $2 a b \leq a^{2}+b^{2}$ to get

$$
\begin{aligned}
\frac{d}{d t}\left\|\rho^{\frac{1}{2}} \chi_{t}\right\|_{L^{2}(\Omega)}^{2}+\frac{d}{d t}\left\|\lambda^{-\frac{1}{2}} \xi\right\|_{L^{2}(\Omega)}^{2} \leq & \left\|\rho^{\frac{1}{2}} \chi_{t}\right\|_{L^{2}(\Omega)}^{2}+\left\|\rho^{\frac{1}{2}} \boldsymbol{\eta}_{t t}\right\|_{L^{2}(\Omega)}^{2} \\
& +\left\|\lambda^{-\frac{1}{2}} \zeta_{t}\right\|_{L^{2}(\Omega)}^{2}+\left\|\lambda^{-\frac{1}{2}} \xi\right\|_{L^{2}(\Omega)}^{2}
\end{aligned}
$$

Now we can apply Gronwall's inequality and

$$
\begin{aligned}
\left\|\rho^{\frac{1}{2}} \boldsymbol{\chi}_{t}\right\|_{L^{2}(\Omega)}^{2}(t)+\left\|\lambda^{-\frac{1}{2}} \xi\right\|_{L^{2}(\Omega)}^{2}(t) \leq & \left\|\rho^{\frac{1}{2}} \chi_{t}(0)\right\|_{L^{2}(\Omega)}^{2}+\left\|\lambda^{-\frac{1}{2}} \xi(0)\right\|_{L^{2}(\Omega)} \\
& +\int_{0}^{t}\left(\left\|\rho^{\frac{1}{2}} \boldsymbol{\eta}_{t t}\right\|_{L^{2}(\Omega)}^{2}+\left\|\lambda^{-\frac{1}{2}} \zeta_{t}\right\|_{L^{2}(\Omega)}^{2}\right) .
\end{aligned}
$$

If we take the supremum over all $t$ and use the initial conditions we obtain

$$
\left\|\rho^{\frac{1}{2}} \chi_{t}\right\|_{L^{\infty}\left(L^{2}\right)}^{2}+\left\|\lambda^{-\frac{1}{2}} \xi\right\|_{L^{\infty}\left(L^{2}\right)}^{2} \leq\left\|\rho^{\frac{1}{2}} \boldsymbol{\eta}_{t t}\right\|_{L^{2}\left(L^{2}\right)}^{2}+\left\|\lambda^{-\frac{1}{2}} \zeta_{t}\right\|_{L^{2}\left(L^{2}\right)}^{2} .
$$

We complete the proof by using the approximation properties (2.2) and (2.4) of the projections.

We now derive an estimate of the error in the displacement in the $L^{\infty}\left(L^{2}\right)$ norm.

TheOREM 4.2. For $t \geq 0$, let $(\boldsymbol{u}(t), p(t))$ be the solution of problem (3.6)(3.10). Assume that $\boldsymbol{u} \in L^{\infty}\left(L^{2}(\Omega)\right), \boldsymbol{u}_{t} \in L^{2}\left(\boldsymbol{H}^{k}(\Omega)\right), \boldsymbol{u}_{t}(0) \in \boldsymbol{H}^{k}(\Omega)$, and $p \in L^{2}\left(H^{k}(\Omega)\right)$. Then there exists a constant $C$ independent of $h$ such that

$$
\begin{aligned}
& \left\|\rho^{\frac{1}{2}}(\boldsymbol{u}-\boldsymbol{U})\right\|_{L^{\infty}\left(L^{2}\right)} \\
\leq & C h^{k}\left(\left\|\boldsymbol{u}_{t}\right\|_{L^{2}\left(H^{k}\right)}+\left\|\boldsymbol{u}_{t}(0)\right\|_{H^{k}}+\|p\|_{L^{2}\left(H^{k}\right)}\right),
\end{aligned}
$$

where $k$ is associated with the degree of the finite element polynomial.

Proof. We first obtain the same equations (4.2) and (4.3) that arrive after taking into account the definition of the $\Pi_{h}$ and the $\mathcal{P}_{h}$ projections

$$
\begin{aligned}
\left(\rho \boldsymbol{\chi}_{t t}, \boldsymbol{v}\right)+(\xi, \nabla \cdot \boldsymbol{v}) & =\left(\rho \boldsymbol{\eta}_{t t}, \boldsymbol{v}\right) & & \forall \boldsymbol{v} \in \boldsymbol{V}_{h}, \\
\left(\lambda^{-1} \xi, w\right)-(\nabla \cdot \boldsymbol{\chi}, w) & =\left(\lambda^{-1} \zeta, w\right) & & \forall w \in W_{h} .
\end{aligned}
$$

If we integrate (4.10) from 0 to $t$, noting that $\chi_{t}(0)=0$, we obtain

$$
\left(\rho \boldsymbol{\chi}_{t}, \boldsymbol{v}\right)+\left(\int_{0}^{t} \xi, \nabla \cdot \boldsymbol{v}\right)=\left(\rho \boldsymbol{\eta}_{t}, \boldsymbol{v}\right)-\left(\rho \boldsymbol{\eta}_{t}(0), \boldsymbol{v}\right) .
$$

We set $\boldsymbol{v}=\boldsymbol{\chi}, \phi=\int_{0}^{t} \xi(s) d s$, and $w=\phi$. Then (4.12) and (4.11) become

$$
\begin{aligned}
\left(\rho \boldsymbol{\chi}_{t}, \boldsymbol{\chi}\right)+(\phi, \nabla \cdot \boldsymbol{\chi}) & =\left(\rho \boldsymbol{\eta}_{t}, \boldsymbol{\chi}\right)-\left(\rho \boldsymbol{\eta}_{t}(0), \boldsymbol{\chi}\right), \\
\left(\lambda^{-1} \xi, \phi\right)-(\nabla \cdot \boldsymbol{\chi}, \phi) & =\left(\lambda^{-1} \zeta, \phi\right) .
\end{aligned}
$$

Adding (4.13) and (4.14) gives

$$
\left(\rho \boldsymbol{\chi}_{t}, \boldsymbol{\chi}\right)+\left(\lambda^{-1} \xi, \phi\right)=\left(\rho \boldsymbol{\eta}_{t}, \boldsymbol{\chi}\right)-\left(\rho \boldsymbol{\eta}_{t}(0), \boldsymbol{\chi}\right)+\left(\lambda^{-1} \zeta, \phi\right) .
$$

Therefore

$$
\left(\rho \boldsymbol{\chi}_{t}, \boldsymbol{\chi}\right)+\left(\lambda^{-1} \phi_{t}, \phi\right)=\left(\rho \boldsymbol{\eta}_{t}, \boldsymbol{\chi}\right)-\left(\rho \boldsymbol{\eta}_{t}(0), \boldsymbol{\chi}\right)+\left(\lambda^{-1} \zeta, \phi\right)
$$


so that

$$
\frac{1}{2} \frac{d}{d t}\left\|\rho^{\frac{1}{2}} \boldsymbol{\chi}\right\|_{L^{2}(\Omega)}^{2}+\frac{1}{2} \frac{d}{d t}\left\|\lambda^{-\frac{1}{2}} \phi\right\|_{L^{2}(\Omega)}^{2}=\left(\rho \boldsymbol{\eta}_{t}, \boldsymbol{\chi}\right)-\left(\rho \boldsymbol{\eta}_{t}(0), \boldsymbol{\chi}\right)+\left(\lambda^{-1} \zeta, \phi\right) .
$$

Multiplying through by 2 and using the Cauchy-Schwarz inequality gives

$$
\begin{aligned}
\frac{d}{d t}\left\|\rho^{\frac{1}{2}} \chi\right\|_{L^{2}(\Omega)}^{2} & +\frac{d}{d t}\left\|\lambda^{-\frac{1}{2}} \phi\right\|_{L^{2}(\Omega)}^{2} \leq 2\left\|\rho^{\frac{1}{2}} \chi\right\|_{L^{2}(\Omega)}\left\|\rho^{\frac{1}{2}} \boldsymbol{\eta}_{t}\right\|_{L^{2}(\Omega)} \\
& +2\left\|\rho^{\frac{1}{2}} \chi\right\|_{L^{2}(\Omega)}\left\|\rho^{\frac{1}{2}} \boldsymbol{\eta}_{t}(0)\right\|_{L^{2}(\Omega)}+2\left\|\lambda^{-\frac{1}{2}} \zeta\right\|_{L^{2}(\Omega)}\left\|\lambda^{-\frac{1}{2}} \phi\right\|_{L^{2}(\Omega)} .
\end{aligned}
$$

Hence

$$
\begin{aligned}
\frac{d}{d t}\left\|\rho^{\frac{1}{2}} \chi\right\|_{L^{2}(\Omega)}^{2} & +\frac{d}{d t}\left\|\lambda^{-\frac{1}{2}} \phi\right\|_{L^{2}(\Omega)}^{2} \leq 2\left\|\rho^{\frac{1}{2}} \chi\right\|_{L^{2}(\Omega)}^{2}\left\|\rho^{\rho^{\frac{1}{2}}} \boldsymbol{\eta}_{t}\right\|_{L^{2}(\Omega)}^{2} \\
& +\left\|\rho^{\frac{1}{2}} \boldsymbol{\eta}_{t}(0)\right\|_{L^{2}(\Omega)}^{2}+\left\|\lambda^{-\frac{1}{2}} \zeta\right\|_{L^{2}(\Omega)}^{2}+\left\|\lambda^{-\frac{1}{2}} \phi\right\|_{L^{2}(\Omega)}^{2} .
\end{aligned}
$$

We apply Gronwall's inequality to (4.15) and note that $\phi(0)=0$ and $\chi(0)=0$ from (3.24). Then

$$
\begin{aligned}
\left\|\rho^{\frac{1}{2}} \chi\right\|_{L^{2}(\Omega)}^{2}(t)+\left\|\lambda^{-\frac{1}{2}} \phi\right\|_{L^{2}(\Omega)}^{2}(t) & \\
& \leq \int_{0}^{t}\left(\left\|\rho^{\frac{1}{2}} \boldsymbol{\eta}_{s}\right\|_{L^{2}(\Omega)}^{2}+\left\|\rho^{\frac{1}{2}} \boldsymbol{\eta}_{s}(0)\right\|_{L^{2}(\Omega)}^{2}+\left\|\lambda^{-\frac{1}{2}} \zeta\right\|_{L^{2}(\Omega)}^{2}\right) d s
\end{aligned}
$$

and thus

$$
\begin{aligned}
\left\|\rho^{\frac{1}{2}} \chi\right\|_{L^{2}(\Omega)}^{2}(t)+\left\|\lambda^{-\frac{1}{2}} \phi\right\|_{L^{2}(\Omega)}^{2}(t) & \\
& \leq C t\left\|\rho^{\frac{1}{2}} \boldsymbol{\eta}_{t}(0)\right\|_{L^{2}(\Omega)}^{2}+C\left\|\rho^{\frac{1}{2}} \boldsymbol{\eta}_{t}\right\|_{L^{2}\left(L^{2}\right)}^{2}+C\left\|\lambda^{-\frac{1}{2}} \zeta\right\|_{L^{2}\left(L^{2}\right)}^{2}
\end{aligned}
$$

We take the supremum over all $t$ to get

$$
\left\|\rho^{\frac{1}{2}} \chi\right\|_{L^{\infty}\left(L^{2}\right)}^{2} \leq C\left\|\rho^{\frac{1}{2}} \boldsymbol{\eta}_{t}\right\|_{L^{2}\left(L^{2}\right)}^{2}+C\left\|\rho^{\frac{1}{2}} \boldsymbol{\eta}_{t}(0)\right\|_{L^{2}(\Omega)}^{2}+C\left\|\lambda^{-\frac{1}{2}} \zeta\right\|_{L^{2}\left(L^{2}\right)}^{2} .
$$

The final result is obtained by using the approximation properties (2.2) and (2.4).

5. Explicit method. In this section, we define further notation, we formulate the fully discrete mixed finite element scheme, and we analyze the stability and the convergence of the discrete method.

5.1. Notation and scheme. Let $\Delta t>0$ be the time step size and define $t_{i}=i \Delta t$ with $t_{N}=T$. For any function $\phi$ of time, let $\phi^{n}$ denote $\phi\left(t_{n}\right)$. We denote $\phi^{n+\frac{1}{2}}=\left(\phi^{n}+\phi^{n+1}\right) / 2$, and we define the following terms for the discrete temporal derivatives:

$$
\partial_{t} \phi^{n}=\frac{\phi^{n+1}-\phi^{n-1}}{2 \Delta t}, \quad \partial_{t} \phi^{n+\frac{1}{2}}=\frac{\phi^{n+1}-\phi^{n}}{\Delta t}, \quad \partial_{t}^{2} \phi^{n}=\frac{\phi^{n+1}-2 \phi^{n}+\phi^{n-1}}{\Delta t^{2}} .
$$


We easily see that we have

$$
\partial_{t}^{2} \phi^{n}=\frac{\partial_{t} \phi^{n+\frac{1}{2}}-\partial_{t} \phi^{n-\frac{1}{2}}}{\Delta t} \quad \text { and } \quad \partial_{t} \phi^{n}=\frac{\partial_{t} \phi^{n+\frac{1}{2}}+\partial_{t} \phi^{n-\frac{1}{2}}}{2} .
$$

The fully discrete mixed finite element scheme is as follows: find $\left(\boldsymbol{U}^{n+1}, P^{n+1}\right)$ in $V_{h} \times W_{h}$ such that

$$
\begin{aligned}
\left(\boldsymbol{U}^{0}, \boldsymbol{v}\right) & =\left(\Pi_{h} \boldsymbol{u}_{0}, \boldsymbol{v}\right) & & \forall \boldsymbol{v} \in \boldsymbol{V}_{h} \\
\left(P^{0}, w\right) & =\left(p^{0}, w\right) & & \forall w \in W_{h} \\
\left(\rho \frac{2}{\Delta t} \partial_{t} \boldsymbol{U}^{\frac{1}{2}}, \boldsymbol{v}\right)+\left(P^{0}, \nabla \cdot \boldsymbol{v}\right) & =\left(\boldsymbol{f}^{0}+\rho \frac{2}{\Delta t} \Pi_{h} \boldsymbol{u}_{1}, \boldsymbol{v}\right) & & \forall \boldsymbol{v} \in \boldsymbol{V}_{h}, \\
\left(\rho \partial_{t}^{2} \boldsymbol{U}^{n}, \boldsymbol{v}\right)+\left(P^{n}, \nabla \cdot \boldsymbol{v}\right) & =\left(\boldsymbol{f}^{n}, \boldsymbol{v}\right) & & \forall \boldsymbol{v} \in \boldsymbol{V}_{h}, \\
\left(\lambda^{-1} P^{n+1}, w\right)-\left(\nabla \cdot \boldsymbol{U}^{n+1}, w\right) & =0 & & \forall w \in W_{h} .
\end{aligned}
$$

5.2. Stability condition for the discrete problem. We show that the scheme is stable for the Dirichlet (homogeneous) problem, and in particular show that the temporal iterates are bound by the initial data. We consider (5.5) and (5.6) for the homogeneous case,

$$
\begin{aligned}
\left(\rho \partial_{t}^{2} \boldsymbol{U}^{n}, \boldsymbol{v}\right)+\left(P^{n}, \nabla \cdot \boldsymbol{v}\right) & =0 & & \forall \boldsymbol{v} \in \boldsymbol{V}_{h}, \\
\left(\lambda^{-1} P^{n+1}, w\right)-\left(\nabla \cdot \boldsymbol{U}^{n+1}, w\right) & =0 & & \forall w \in W_{h} .
\end{aligned}
$$

As in [7], we use the "inverse assumption," which states that there exists a constant $C_{0}$, independent of $h$, such that

$$
\|\nabla \cdot \phi\|_{L^{2}(\Omega)} \leq C_{0} h^{-1}\|\phi\|_{L^{2}(\Omega)}
$$

for $\phi \in W_{h}$.

THEOREM 5.1. The explicit scheme defined by (5.2)-(5.6) is stable if $\Delta t<\frac{2 h \rho_{0}^{\frac{1}{2}}}{C_{0} \lambda_{1}^{\frac{1}{2}}}$.

That is,

$$
\left(1-\frac{\Delta t^{2} C_{0}^{2} \lambda_{1}}{4 h^{2} \rho_{0}}\right)\left\|\partial_{t} \boldsymbol{U}^{N+\frac{1}{2}}\right\|_{L^{2}(\Omega)}^{2}+\left\|P^{N+\frac{1}{2}}\right\|_{L^{2}(\Omega)}^{2}
$$

is bound by initial data.

Proof. If we subtract (5.8) from itself, with $n+1$ replaced by $n-1$, we get

$$
\left(\lambda^{-1}\left(P^{n+1}-P^{n-1}\right), w\right)-\left(\nabla \cdot\left(\boldsymbol{U}^{n+1}-\boldsymbol{U}^{n-1}\right), w\right)=0, \quad w \in W_{h} .
$$

As (5.7) holds for all $\boldsymbol{v} \in \boldsymbol{V}_{h}$ and (5.11) holds for all $w \in W_{h}$, we set $\boldsymbol{v}=\partial_{t} \boldsymbol{U}^{n}$ and $w=\frac{P^{n}}{2 \Delta t}$. This gives

$$
\begin{aligned}
\left(\rho \partial_{t}^{2} \boldsymbol{U}^{n}, \partial_{t} \boldsymbol{U}^{n}\right)+\left(P^{n}, \nabla \cdot \partial_{t} \boldsymbol{U}^{n}\right) & =0 \\
\left(\lambda^{-1}\left(P^{n+1}-P^{n-1}\right), \frac{P^{n}}{2 \Delta t}\right)-\left(\nabla \cdot\left(\boldsymbol{U}^{n+1}-\boldsymbol{U}^{n-1}\right), \frac{P^{n}}{2 \Delta t}\right) & =0 .
\end{aligned}
$$

If we add (5.12) and (5.13), we obtain

$$
\left(\rho \partial_{t}^{2} \boldsymbol{U}^{n}, \frac{\boldsymbol{U}^{n+1}-\boldsymbol{U}^{n-1}}{2 \Delta t}\right)+\left(\lambda^{-1}\left(P^{n+1}-P^{n-1}\right), \frac{P^{n}}{2 \Delta t}\right)=0 .
$$


Substituting (5.1) into (5.14) above yields

$$
\begin{aligned}
& \frac{1}{2 \Delta t}\left(\rho\left(\partial_{t} \boldsymbol{U}^{n+\frac{1}{2}}-\partial_{t} \boldsymbol{U}^{n-\frac{1}{2}}\right), \partial_{t} \boldsymbol{U}^{n+\frac{1}{2}}+\partial_{t} \boldsymbol{U}^{n-\frac{1}{2}}\right)+\left(\lambda^{-1} \partial_{t} P^{n}, P^{n}\right) \\
& \quad=\frac{1}{2 \Delta t}\left(\left\|\rho^{\frac{1}{2}} \partial_{t} \boldsymbol{U}^{n+\frac{1}{2}}\right\|_{L^{2}(\Omega)}^{2}-\left\|\rho^{\frac{1}{2}} \partial_{t} \boldsymbol{U}^{n-\frac{1}{2}}\right\|_{L^{2}(\Omega)}^{2}\right)+\left(\lambda^{-1} \partial_{t} P^{n}, P^{n}\right)=0 .
\end{aligned}
$$

We now examine $\left(\partial_{t} P^{n}, P^{n}\right)$. We have

$$
\partial_{t} P^{n}=\frac{P^{n+1}+P^{n}-P^{n}-P^{n-1}}{2 \Delta t}=\frac{\left(P^{n+\frac{1}{2}}-P^{n-\frac{1}{2}}\right)}{\Delta t} .
$$

We can rewrite $P^{n}$ as

$$
\begin{aligned}
P^{n} & =\frac{P^{n}+P^{n+1}}{4}+\frac{P^{n-1}+P^{n}}{4}-\frac{\Delta t^{2}}{4}\left(\frac{P^{n+1}-2 P^{n}+P^{n-1}}{\Delta t^{2}}\right) \\
& =\frac{P^{n}+P^{n+1}}{4}+\frac{P^{n-1}+P^{n}}{4}-\frac{\Delta t^{2}}{4} \partial_{t}^{2} P^{n} \\
& =\frac{P^{n+\frac{1}{2}}}{2}+\frac{P^{n-\frac{1}{2}}}{2}-\frac{\Delta t^{2}}{4} \partial_{t}^{2} P^{n} .
\end{aligned}
$$

If we use (5.16) and (5.17) in $\left(\partial_{t} P^{n}, P^{n}\right)$, we have

$$
\begin{aligned}
\left(\lambda^{-1} \partial_{t} P^{n}, P^{n}\right)= & \frac{1}{2 \Delta t}\left(\lambda^{-1}\left(P^{n+\frac{1}{2}}-P^{n-\frac{1}{2}}\right), P^{n+\frac{1}{2}}+P^{n-\frac{1}{2}}\right) \\
& -\left(\lambda^{-1} \partial_{t} P^{n}, \frac{\Delta t^{2}}{4} \partial_{t}^{2} P^{n}\right) \\
= & \frac{1}{2 \Delta t}\left(\lambda^{-1}\left(P^{n+\frac{1}{2}}-P^{n-\frac{1}{2}}\right), P^{n+\frac{1}{2}}+P^{n-\frac{1}{2}}\right) \\
& -\left(\frac{\lambda^{-1}}{2}\left(\partial_{t} P^{n+\frac{1}{2}}+\partial_{t} P^{n-\frac{1}{2}}\right), \frac{\Delta t^{2}}{4} \frac{\left(\partial_{t} P^{n+\frac{1}{2}}-\partial_{t} P^{n-\frac{1}{2}}\right)}{\Delta t}\right) \\
= & \frac{1}{2 \Delta t}\left[\left\|\lambda^{-\frac{1}{2}} P^{n+\frac{1}{2}}\right\|_{L^{2}(\Omega)}^{2}-\left\|\lambda^{-\frac{1}{2}} P^{n-\frac{1}{2}}\right\|_{L^{2}(\Omega)}^{2}\right. \\
& \left.\quad-\frac{\Delta t^{2}}{4}\left(\left\|\lambda^{-\frac{1}{2}} \partial_{t} P^{n+\frac{1}{2}}\right\|_{L^{2}(\Omega)}^{2}-\left\|\lambda^{-\frac{1}{2}} \partial_{t} P^{n-\frac{1}{2}}\right\|_{L^{2}(\Omega)}^{2}\right)\right] .
\end{aligned}
$$

Thus (5.15) becomes

$$
\begin{aligned}
\left\|\rho^{\frac{1}{2}} \partial_{t} \boldsymbol{U}^{n+\frac{1}{2}}\right\|_{L^{2}(\Omega)}^{2} & -\left\|\rho^{\frac{1}{2}} \partial_{t} \boldsymbol{U}^{n-\frac{1}{2}}\right\|_{L^{2}(\Omega)}^{2} \\
& +\left\|\lambda^{-\frac{1}{2}} P^{n+\frac{1}{2}}\right\|_{L^{2}(\Omega)}^{2}-\left\|\lambda^{-\frac{1}{2}} P^{n-\frac{1}{2}}\right\|_{L^{2}(\Omega)}^{2} \\
& -\frac{\Delta t^{2}}{4}\left(\left\|\lambda^{-\frac{1}{2}} \partial_{t} P^{n+\frac{1}{2}}\right\|_{L^{2}(\Omega)}^{2}-\left\|\lambda^{-\frac{1}{2}} \partial_{t} P^{n-\frac{1}{2}}\right\|_{L^{2}(\Omega)}^{2}\right)=0
\end{aligned}
$$

If we sum (5.18) from $n=1, \ldots, N$, we get

$$
\begin{aligned}
\left\|\rho^{\frac{1}{2}} \partial_{t} \boldsymbol{U}^{N+\frac{1}{2}}\right\|_{L^{2}(\Omega)}^{2} & +\left\|\lambda^{-\frac{1}{2}} P^{N+\frac{1}{2}}\right\|_{L^{2}(\Omega)}^{2}-\frac{\Delta t^{2}}{4}\left\|\lambda^{-\frac{1}{2}} \partial_{t} P^{N+\frac{1}{2}}\right\|_{L^{2}(\Omega)}^{2} \\
& =\left\|\rho^{\frac{1}{2}} \partial_{t} \boldsymbol{U}^{\frac{1}{2}}\right\|_{L^{2}(\Omega)}^{2}+\left\|\lambda^{-\frac{1}{2}} P^{\frac{1}{2}}\right\|_{L^{2}(\Omega)}^{2}-\frac{\Delta t^{2}}{4}\left\|\lambda^{-\frac{1}{2}} \partial_{t} P^{\frac{1}{2}}\right\|_{L^{2}(\Omega)}^{2} .
\end{aligned}
$$


We recall from (5.11) that

$$
\left(\lambda^{-1}\left(P^{n+1}-P^{n}\right), w\right)-\left(\nabla \cdot\left(\boldsymbol{U}^{n+1}-\boldsymbol{U}^{n}\right), w\right)=0 .
$$

Then, by the Cauchy-Schwarz inequality and (5.9),

$$
\begin{aligned}
\left(\lambda^{-\frac{1}{2}}\left(P^{N+1}-P^{N}\right), w\right) & =\left(\nabla \cdot\left(\boldsymbol{U}^{N+1}-\boldsymbol{U}^{N}\right), w\right) \\
& \leq\left\|\nabla \cdot\left(\boldsymbol{U}^{N+1}-\boldsymbol{U}^{N}\right)\right\|_{L^{2}(\Omega)}\|w\|_{L^{2}(\Omega)} \\
& \leq \frac{C_{0}}{h}\left\|\boldsymbol{U}^{N+1}-\boldsymbol{U}^{N}\right\|_{L^{2}(\Omega)}\|w\|_{L^{2}(\Omega)} \\
& \leq \frac{C_{0}}{h}(\Delta t)\left\|\partial_{t} \boldsymbol{U}^{N+\frac{1}{2}}\right\|_{L^{2}(\Omega)}\|w\|_{L^{2}(\Omega)} .
\end{aligned}
$$

Thus

$$
(\Delta t)\left(\lambda^{-\frac{1}{2}} \partial_{t} P^{N+\frac{1}{2}}, w\right) \leq \frac{C_{0}}{h}(\Delta t)\left\|\partial_{t} \boldsymbol{U}^{N+\frac{1}{2}}\right\|_{L^{2}(\Omega)}\|w\|_{L^{2}(\Omega)} .
$$

We choose $w=\partial_{t} P^{N+\frac{1}{2}}$, so

$$
\begin{aligned}
\left\|\lambda^{-\frac{1}{2}} \partial_{t} P^{N+\frac{1}{2}}\right\|_{L^{2}(\Omega)}^{2} & \leq \frac{C_{0}}{h}\left\|\partial_{t} \boldsymbol{U}^{N+\frac{1}{2}}\right\|_{L^{2}(\Omega)}\left\|\partial_{t} P^{N+\frac{1}{2}}\right\|_{L^{2}(\Omega)} \\
& \leq \frac{C_{0} \lambda_{1}^{\frac{1}{2}}}{h \rho_{0}^{\frac{1}{2}}}\left\|\rho^{\frac{1}{2}} \partial_{t} \boldsymbol{U}^{N+\frac{1}{2}}\right\|_{L^{2}(\Omega)}\left\|\lambda^{-\frac{1}{2}} \partial_{t} P^{N+\frac{1}{2}}\right\|_{L^{2}(\Omega)}
\end{aligned}
$$

or

$$
\left\|\lambda^{-\frac{1}{2}} \partial_{t} P^{N+\frac{1}{2}}\right\|_{L^{2}(\Omega)} \leq \frac{C_{0} \lambda_{1}^{\frac{1}{2}}}{h \rho_{0}^{\frac{1}{2}}}\left\|\rho^{\frac{1}{2}} \partial_{t} \boldsymbol{U}^{N+\frac{1}{2}}\right\|_{L^{2}(\Omega)}
$$

Hence

$$
\begin{gathered}
\left\|\rho^{\frac{1}{2}} \partial_{t} \boldsymbol{U}^{\frac{1}{2}}\right\|_{L^{2}(\Omega)}^{2}+\left\|\lambda^{-\frac{1}{2}} P^{\frac{1}{2}}\right\|_{L^{2}(\Omega)}^{2}-\frac{\Delta t^{2}}{4}\left\|\lambda^{-\frac{1}{2}} \partial_{t} P^{\frac{1}{2}}\right\|_{L^{2}(\Omega)}^{2} \\
=\left\|\rho^{\frac{1}{2}} \partial_{t} \boldsymbol{U}^{N+\frac{1}{2}}\right\|_{L^{2}(\Omega)}^{2}+\left\|\lambda^{-\frac{1}{2}} P^{N+\frac{1}{2}}\right\|_{L^{2}(\Omega)}^{2} \\
-\frac{\Delta t^{2}}{4}\left\|\lambda^{-\frac{1}{2}} \partial_{t} P^{N+\frac{1}{2}}\right\|_{L^{2}(\Omega)}^{2} \\
\geq\left\|\rho^{\frac{1}{2}} \partial_{t} \boldsymbol{U}^{N+\frac{1}{2}}\right\|_{L^{2}(\Omega)}^{2}+\left\|\lambda^{-\frac{1}{2}} P^{N+\frac{1}{2}}\right\|_{L^{2}(\Omega)}^{2} \\
-\frac{\Delta t^{2} C_{0}^{2} \lambda_{1}}{4 h^{2} \rho_{0}}\left\|\rho^{\frac{1}{2}} \partial_{t} \boldsymbol{U}^{N+\frac{1}{2}}\right\|_{L^{2}(\Omega)}^{2} \\
=\left(1-\frac{\Delta t^{2} C_{0}^{2} \lambda_{1}}{4 h^{2} \rho_{0}}\right)\left\|\rho^{\frac{1}{2}} \partial_{t} \boldsymbol{U}^{N+\frac{1}{2}}\right\|_{L^{2}(\Omega)}^{2} \\
+\left\|\lambda^{-\frac{1}{2}} P^{N+\frac{1}{2}}\right\|_{L^{2}(\Omega)}^{2} .
\end{gathered}
$$

Thus the temporal iterates are bound by the initial data and the discrete in time scheme is stable if $\Delta t<\frac{2 h \rho_{0}^{\frac{1}{2}}}{\lambda_{1}^{\frac{1}{2}} C_{0}}$. 
5.3. Discrete in time a priori error estimates. We now can derive the estimates for the fully discrete scheme.

TheOREM 5.2. If $\boldsymbol{u} \in L^{\infty}(\boldsymbol{H}(\Omega ; \operatorname{div})), \frac{\partial^{3} \boldsymbol{u}}{\partial t^{3}} \in L^{1}\left(\boldsymbol{L}^{2}(\Omega)\right) \frac{\partial^{4} \boldsymbol{u}}{\partial t^{4}} \in L^{\infty}\left(\boldsymbol{L}^{2}(\Omega)\right)$, and $p \in L^{\infty}\left(L^{2}(\Omega)\right)$, then for $\left\{\boldsymbol{U}^{n}, P^{n}\right\}$ defined by (5.3)-(5.6) there exists a constant $C$ independent of $h$ and $\Delta t$ such that if $\Delta t<\frac{2 h \rho_{0}^{\frac{1}{2}}}{\lambda_{1}^{\frac{1}{2}} C_{0}}$, then

$$
\begin{aligned}
\| \rho^{\frac{1}{2}}(\boldsymbol{u}-\boldsymbol{U}) & \left\|_{l^{\infty}\left(L^{2}\right)}+\right\| \lambda^{\frac{1}{2}}(p-P) \|_{l^{\infty}\left(L^{2}\right)} \\
& \leq C\left(h^{k}+\Delta t^{2}\right)\left(\|\boldsymbol{u}\|_{L^{\infty}\left(H^{k}\right)}+\left\|\frac{\partial^{3} \boldsymbol{u}}{\partial t^{3}}\right\|_{L^{\infty}\left(L^{2}\right)}+\|p\|_{L^{\infty}\left(L^{2}\right)}\right),
\end{aligned}
$$

where $k$ is associated with the degree of the finite element polynomial.

Proof. From (3.9)-(3.10) and (5.5)-(5.6), and by the properties of the $L^{2}$ and $\Pi_{h}$ projections, we can write

$$
\begin{aligned}
\left(\rho \partial_{t}^{2} \boldsymbol{\chi}^{n}, \boldsymbol{v}\right)+\left(\xi^{n}, \nabla \cdot \boldsymbol{v}\right) & =\left(\rho \partial_{t}^{2} \boldsymbol{\eta}^{n}, \boldsymbol{v}\right)+\left(\boldsymbol{r}^{n}, \boldsymbol{v}\right), \\
\left(\lambda^{-1} \xi^{n+1}, w\right)-\left(\nabla \cdot \chi^{n+1}, w\right) & =\left(\lambda^{-1} \zeta^{n+1}, w\right),
\end{aligned}
$$

where $\boldsymbol{r}^{n}=\rho\left(\frac{\partial^{2} \boldsymbol{u}}{\partial t^{2}}\left(t^{n}\right)-\partial_{t}^{2} \boldsymbol{u}^{n}\right)$. We introduce

$$
\phi^{0}=\frac{\Delta t}{2} \xi^{0}, \quad \phi^{n}=\frac{\Delta t}{2} \xi^{0}+\Delta t \sum_{i=1}^{n} \xi^{i} .
$$

Using (5.1) in (5.20) gives

$$
\left(\rho \frac{\partial_{t} \boldsymbol{\chi}^{n+\frac{1}{2}}-\partial_{t} \boldsymbol{\chi}^{n-\frac{1}{2}}}{\Delta t}, \boldsymbol{v}\right)+\left(\xi^{n}, \nabla \cdot \boldsymbol{v}\right)=\left(\rho \frac{\partial_{t} \boldsymbol{\eta}^{n+\frac{1}{2}}-\partial_{t} \boldsymbol{\eta}^{n-\frac{1}{2}}}{\Delta t}, \boldsymbol{v}\right)+\left(\boldsymbol{r}^{n}, \boldsymbol{v}\right) .
$$

Summing over time levels and multiplying through by $\Delta t$ gives

$$
\begin{aligned}
\left(\rho\left(\partial_{t} \chi^{n+\frac{1}{2}}-\partial_{t} \chi^{\frac{1}{2}}\right), \boldsymbol{v}\right)+ & \left(\phi^{n}-\phi^{0}, \nabla \cdot \boldsymbol{v}\right) \\
& =\left(\rho\left(\partial_{t} \boldsymbol{\eta}^{n+\frac{1}{2}}-\partial_{t} \boldsymbol{\eta}^{\frac{1}{2}}\right), \boldsymbol{v}\right)+\left(\Delta t \sum_{i=1}^{n} \boldsymbol{r}^{i}, \boldsymbol{v}\right),
\end{aligned}
$$

since $\Delta t \sum_{i=1}^{n} \xi^{i}=\phi^{n}-\phi^{0}$.

Using (5.4), we obtain

$$
\begin{aligned}
\left(\rho \partial_{t} \boldsymbol{\chi}^{\frac{1}{2}}, \boldsymbol{v}\right) & +\frac{\Delta t}{2}\left(\xi^{0}, \nabla \cdot \boldsymbol{v}\right) \\
& =\left(\rho \partial_{t} \boldsymbol{\eta}^{\frac{1}{2}}, \boldsymbol{v}\right)+\left(\rho\left(\Pi \boldsymbol{u}_{1}-\boldsymbol{u}_{1}\right), \boldsymbol{v}\right)-\frac{1}{2 \Delta t} \int_{0}^{\Delta t} \rho(\Delta t-t)^{2}\left(\frac{\partial^{3} \boldsymbol{u}}{\partial t^{3}}, \boldsymbol{v}\right) d t
\end{aligned}
$$

and thus (5.22) reduces to

$$
\left(\rho \partial_{t} \chi^{n+\frac{1}{2}}, \boldsymbol{v}\right)+\left(\phi^{n}, \nabla \cdot \boldsymbol{v}\right)=\left(\rho \partial_{t} \boldsymbol{\eta}^{n+\frac{1}{2}}, \boldsymbol{v}\right)+\left(\boldsymbol{R}^{n}, \boldsymbol{v}\right),
$$


where $\boldsymbol{R}^{n}$ is defined as

$$
\boldsymbol{R}^{n}=\Delta t \sum_{i=1}^{n} \boldsymbol{r}^{i}+\rho\left(\Pi_{h} \boldsymbol{u}_{1}-\boldsymbol{u}_{1}\right)-\frac{1}{2 \Delta t} \int_{0}^{\Delta t} \rho(\Delta t-t)^{2} \frac{\partial^{3} \boldsymbol{u}}{\partial t^{3}}(t) d t .
$$

We now rewrite (5.21) by noting that $\xi^{n+1}=\partial_{t} \phi^{n+\frac{1}{2}}$, so that

$$
\left(\lambda^{-1} \partial_{t} \phi^{n+\frac{1}{2}}, w\right)-\left(\nabla \cdot \chi^{n+1}, w\right)=\left(\lambda^{-1} \zeta^{n+1}, w\right)
$$

We choose $\boldsymbol{v}=\chi^{n+\frac{1}{2}}$ and $w=\phi^{n+\frac{1}{2}}$, which, when substituted into (5.23) and (5.24), gives

$$
\begin{aligned}
& \left(\rho \partial_{t} \chi^{n+\frac{1}{2}}, \chi^{n+\frac{1}{2}}\right)+\left(\phi^{n}, \nabla \cdot \chi^{n+\frac{1}{2}}\right)=\left(\rho \partial_{t} \boldsymbol{\eta}^{n+\frac{1}{2}}, \chi^{n+\frac{1}{2}}\right)+\left(\boldsymbol{R}^{n}, \chi^{n+\frac{1}{2}}\right), \\
& \left(\lambda^{-1} \partial_{t} \phi^{n+\frac{1}{2}}, \phi^{n+\frac{1}{2}}\right)-\left(\nabla \cdot \chi^{n+1}, \phi^{n+\frac{1}{2}}\right)=\left(\lambda^{-1} \zeta^{n+1}, \phi^{n+\frac{1}{2}}\right) .
\end{aligned}
$$

We expand (5.25) and (5.26) to get

$$
\begin{gathered}
\left(\rho\left(\frac{\chi^{n+1}-\chi^{n}}{\Delta t}\right), \frac{\chi^{n+1}+\chi^{n}}{2}\right)+\left(\phi^{n}, \frac{\nabla \cdot\left(\chi^{n+1}+\chi^{n}\right)}{2}\right) \\
=\left(\rho \partial_{t} \boldsymbol{\eta}^{n+\frac{1}{2}}, \chi^{n+\frac{1}{2}}\right)+\left(\boldsymbol{R}^{n}, \chi^{n+\frac{1}{2}}\right) \\
\left(\lambda^{-1}\left(\frac{\phi^{n+1}-\phi^{n}}{\Delta t}\right), \frac{\phi^{n+1}+\phi^{n}}{2}\right)-\left(\nabla \cdot \chi^{n+1}, \frac{\phi^{n+1}+\phi^{n}}{2}\right)=\left(\lambda^{-1} \zeta^{n+1}, \phi^{n+\frac{1}{2}}\right),
\end{gathered}
$$

so that

$$
\begin{aligned}
\frac{1}{2 \Delta t}\left(\left\|\rho^{\frac{1}{2}} \chi^{n+1}\right\|_{L^{2}(\Omega)}^{2}-\left\|\rho^{\frac{1}{2}} \chi^{n}\right\|_{L^{2}(\Omega)}^{2}\right) & +\frac{1}{2}\left(\phi^{n}, \nabla \cdot \boldsymbol{\chi}^{n+1}\right)+\frac{1}{2}\left(\phi^{n}, \nabla \cdot \boldsymbol{\chi}^{n}\right) \\
& =\left(\rho \partial_{t} \boldsymbol{\eta}^{n+\frac{1}{2}}, \boldsymbol{\chi}^{n+\frac{1}{2}}\right)+\left(\boldsymbol{R}^{n}, \boldsymbol{\chi}^{n+\frac{1}{2}}\right) \\
\frac{1}{2 \Delta t}\left(\left\|\lambda^{-\frac{1}{2}} \phi^{n+1}\right\|_{L^{2}(\Omega)}^{2}-\left\|\lambda^{-\frac{1}{2}} \phi^{n}\right\|_{L^{2}(\Omega)}^{2}\right) & =\frac{1}{2}\left(\nabla \cdot \chi^{n+1}, \phi^{n}\right)+\frac{1}{2}\left(\nabla \cdot \chi^{n+1}, \phi^{n+1}\right) \\
& +\left(\lambda^{-1} \zeta^{n+1}, \phi^{n+\frac{1}{2}}\right) .
\end{aligned}
$$

Adding (5.27) and (5.28) and multiplying by $2 \Delta t$ gives

$$
\begin{aligned}
&\left\|\rho^{\frac{1}{2}} \chi^{n+1}\right\|_{L^{2}(\Omega)}^{2}-\left\|\rho^{\frac{1}{2}} \chi^{n}\right\|_{L^{2}(\Omega)}^{2}+\left\|\lambda^{-\frac{1}{2}} \phi^{n+1}\right\|_{L^{2}(\Omega)}^{2}-\left\|\lambda^{-\frac{1}{2}} \phi^{n}\right\|_{L^{2}(\Omega)}^{2} \\
&+\Delta t\left[\left(\phi^{n}, \nabla \cdot \chi^{n}\right)-\left(\nabla \cdot \chi^{n+1}, \phi^{n+1}\right)\right] \\
&=2 \Delta t\left[\left(\rho \partial_{t} \boldsymbol{\eta}^{n+\frac{1}{2}}, \chi^{n+\frac{1}{2}}\right)+\left(\boldsymbol{R}^{n}, \boldsymbol{\chi}^{n+\frac{1}{2}}\right)\right]+2 \Delta t\left(\lambda^{-1} \zeta^{n+1}, \phi^{n+\frac{1}{2}}\right) .
\end{aligned}
$$

The terms on the right-hand side are bound using the Cauchy-Schwarz inequality as

$$
\begin{aligned}
\left(\rho \partial_{t} \boldsymbol{\eta}^{n+\frac{1}{2}}, \boldsymbol{\chi}^{n+\frac{1}{2}}\right) & \leq\left\|\rho^{\frac{1}{2}} \partial_{t} \boldsymbol{\eta}^{n+\frac{1}{2}}\right\|_{L^{2}(\Omega)}\left\|\chi^{n+\frac{1}{2}}\right\|_{L^{2}(\Omega)} \\
\left(\boldsymbol{R}^{n}, \boldsymbol{\chi}^{n+\frac{1}{2}}\right) & \leq\left\|\boldsymbol{R}^{n}\right\|_{L^{2}(\Omega)}\left\|\chi^{n+\frac{1}{2}}\right\|_{L^{2}(\Omega)} \\
\left(\lambda^{-1} \zeta^{n+1}, \phi^{n+\frac{1}{2}}\right) & \leq\left\|\lambda^{-\frac{1}{2}} \zeta^{n+1}\right\|_{L^{2}(\Omega)}\left\|\lambda^{-\frac{1}{2}} \phi^{n+\frac{1}{2}}\right\|_{L^{2}(\Omega)} .
\end{aligned}
$$


Sum (5.29) over time levels to get

$$
\begin{aligned}
& \left\|\rho^{\frac{1}{2}} \chi^{n+1}\right\|_{L^{2}(\Omega)}^{2}-\left\|\rho^{\frac{1}{2}} \chi^{0}\right\|_{L^{2}(\Omega)}^{2}+\left\|\lambda^{-\frac{1}{2}} \phi^{n+1}\right\|_{L^{2}(\Omega)}^{2} \\
& -\left\|\lambda^{-\frac{1}{2}} \phi^{0}\right\|_{L^{2}(\Omega)}^{2}-\Delta t\left[\left(\nabla \cdot \chi^{n+1}, \phi^{n+1}\right)-\left(\phi^{0}, \nabla \cdot \chi^{0}\right)\right] \\
& \leq 2 \Delta t \sum_{i=0}^{n}\left(\left\|\rho^{\frac{1}{2}} \partial_{t} \eta^{i+\frac{1}{2}}\right\|_{L^{2}(\Omega)}\left\|\chi^{i+\frac{1}{2}}\right\|_{L^{2}(\Omega)}+\left\|R^{i}\right\|_{L^{2}(\Omega)}\left\|\chi^{i+\frac{1}{2}}\right\|_{L^{2}(\Omega)}\right) \\
& \quad+\Delta t \sum_{i=0}^{n}\left(\left\|\lambda^{-\frac{1}{2}} \zeta^{i+1}\right\|_{L^{2}(\Omega)}\left(\left\|\lambda^{-\frac{1}{2}} \phi^{i+1}\right\|_{L^{2}(\Omega)}+\left\|\lambda^{-\frac{1}{2}} \phi^{i}\right\|_{L^{2}(\Omega)}\right)\right) .
\end{aligned}
$$

After imposing the initial conditions (5.2) and (5.3) in (5.30) we have

$$
\begin{aligned}
& \left\|\rho^{\frac{1}{2}} \chi^{n+1}\right\|_{L^{2}(\Omega)}^{2}+\left\|\lambda^{-\frac{1}{2}} \phi^{n+1}\right\|_{L^{2}(\Omega)}^{2}-\Delta t\left(\nabla \cdot \chi^{n+1}, \phi^{n+1}\right) \\
& \leq 2 \Delta t \sum_{i=0}^{n}\left(\left\|\rho^{\frac{1}{2}} \partial_{t} \boldsymbol{\eta}^{i+\frac{1}{2}}\right\|_{L^{2}(\Omega)}\left\|\chi^{i+\frac{1}{2}}\right\|_{L^{2}(\Omega)}+\left\|\boldsymbol{R}^{i}\right\|_{L^{2}(\Omega)}\left\|\chi^{i+\frac{1}{2}}\right\|_{L^{2}(\Omega)}\right) \\
& \quad+\Delta t \sum_{i=0}^{n}\left(\left\|\lambda^{-\frac{1}{2}} \zeta^{i+1}\right\|_{L^{2}(\Omega)}\left(\left\|\lambda^{-\frac{1}{2}} \phi^{i+1}\right\|_{L^{2}(\Omega)}+\left\|\lambda^{-\frac{1}{2}} \phi^{i}\right\|_{L^{2}(\Omega)}\right)\right) .
\end{aligned}
$$

Using the Cauchy-Schwarz inequality and the inverse assumption (5.9) and choosing $h$ and $\Delta t$ such that $\Delta t<\frac{2 h \rho_{0}^{\frac{1}{2}}}{C_{0} \lambda_{1}^{\frac{1}{2}}}$, we have that

$$
\begin{aligned}
\Delta t\left(\nabla \cdot \chi^{n+1}, \phi^{n+1}\right) & \leq \Delta t\left\|\nabla \cdot \chi^{n+1}\right\|_{L^{2}(\Omega)}\left\|\phi^{n+1}\right\|_{L^{2}(\Omega)} \\
& \leq \Delta t C_{0} h^{-1}\left\|\chi^{n+1}\right\|_{L^{2}(\Omega)}\left\|\phi^{n+1}\right\|_{L^{2}(\Omega)} \\
& \leq \frac{\Delta t C_{0} \lambda_{1}^{\frac{1}{2}}}{2 h \rho_{0}^{\frac{1}{2}}}\left(\left\|\rho^{\frac{1}{2}} \chi^{n+1}\right\|_{L^{2}(\Omega)}^{2}+\left\|\lambda^{-\frac{1}{2}} \phi^{n+1}\right\|_{L^{2}(\Omega)}^{2}\right) \\
& <\left\|\rho^{\frac{1}{2}} \chi^{n+1}\right\|_{L^{2}(\Omega)}^{2}+\left\|\lambda^{-\frac{1}{2}} \phi^{n+1}\right\|_{L^{2}(\Omega)}^{2} .
\end{aligned}
$$

Thus we have

$$
\begin{array}{r}
\left\|\rho^{\frac{1}{2}} \chi^{n+1}\right\|_{L^{2}(\Omega)}^{2}+\left\|\lambda^{-\frac{1}{2}} \phi^{n+1}\right\|_{L^{2}(\Omega)}^{2} \\
\leq C \Delta t \sum_{i=0}^{n}\left(\left\|\rho^{\frac{1}{2}} \partial_{t} \eta^{i+\frac{1}{2}}\right\|_{L^{2}(\Omega)}\left\|\chi^{i+\frac{1}{2}}\right\|_{L^{2}(\Omega)}+\left\|\boldsymbol{R}^{i}\right\|_{L^{2}(\Omega)}\left\|\chi^{i+\frac{1}{2}}\right\|_{L^{2}(\Omega)}\right) \\
+2\left\|\lambda^{-\frac{1}{2}} \phi\right\|_{l^{\infty}\left(L^{2}\right)}\left(\Delta t \sum_{i=0}^{n}\left\|\lambda^{-\frac{1}{2}} \zeta^{i+1}\right\|_{L^{2}(\Omega)}\right) .
\end{array}
$$


Since $\left\|\chi^{i+\frac{1}{2}}\right\|_{L^{2}(\Omega)} \leq\|\chi\|_{l^{\infty}\left(L^{2}\right)}$, then

$$
\begin{gathered}
\left\|\rho^{\frac{1}{2}} \chi^{n+1}\right\|_{L^{2}(\Omega)}^{2}+\left\|\lambda^{-\frac{1}{2}} \phi^{n+1}\right\|_{L^{2}(\Omega)}^{2} \\
\leq \frac{C 2 \Delta t}{\rho_{0}^{\frac{1}{2}}}\left\|\rho^{\frac{1}{2}} \chi\right\|_{l^{\infty}\left(L^{2}\right)}\left(\sum_{i=0}^{n}\left\|\rho^{\frac{1}{2}} \partial_{t} \boldsymbol{\eta}^{i+\frac{1}{2}}\right\|_{L^{2}(\Omega)}+\sum_{i=0}^{n}\left\|\boldsymbol{R}^{i}\right\|_{L^{2}(\Omega)}\right) \\
+\frac{1}{4}\left\|\lambda^{-\frac{1}{2}} \phi\right\|_{l^{\infty}\left(L^{2}\right)}^{2}+C\left(\Delta t \sum_{i=0}^{n}\left\|\lambda^{-\frac{1}{2}} \zeta^{i+1}\right\|_{L^{2}(\Omega)}\right)^{2} \\
\leq \frac{1}{4}\left\|\rho^{\frac{1}{2}} \chi\right\|_{l^{\infty}\left(L^{2}\right)}^{2}+C \Delta t^{2}\left(\sum_{i=0}^{N}\left\|\rho^{\frac{1}{2}} \partial_{t} \boldsymbol{\eta}^{i+\frac{1}{2}}\right\|{ }_{L^{2}(\Omega)}\right)^{2} \\
+C \Delta t^{2}\left(\sum_{i=0}^{N}\left\|\boldsymbol{R}^{i}\right\|_{L^{2}(\Omega)}\right)^{2}+\frac{1}{4}\left\|\lambda^{-\frac{1}{2}} \phi\right\|_{l^{\infty}\left(L^{2}\right)}^{2} \\
+C\left(\Delta t \sum_{i=0}^{n}\left\|\lambda^{-\frac{1}{2}} \zeta^{i+1}\right\|_{L^{2}(\Omega)}\right)^{2} .
\end{gathered}
$$

If we take the supremum on $n$ on the left-hand side we get

$$
\begin{aligned}
\left\|\rho^{\frac{1}{2}} \chi\right\|_{l^{\infty}\left(L^{2}\right)}^{2} & +\left\|\lambda^{-\frac{1}{2}} \phi\right\|_{l^{\infty}\left(L^{2}\right)}^{2} \leq C\left\|\lambda^{-\frac{1}{2}} \zeta\right\|_{l^{\infty}\left(L^{2}\right)}^{2} \\
& +C \Delta t^{2}\left(\sum_{i=0}^{N}\left\|\rho^{\frac{1}{2}} \partial_{t} \boldsymbol{\eta}^{i+\frac{1}{2}}\right\|_{L^{2}(\Omega)}\right)^{2}+C \Delta t^{2}\left(\sum_{i=0}^{N}\left\|\boldsymbol{R}^{i}\right\|_{L^{2}(\Omega)}\right)^{2} .
\end{aligned}
$$

The first two terms in the right-hand side of the previous inequality can be bound using the approximation properties and the bound

$$
\Delta t \sum_{i=0}^{N}\left\|\rho^{\frac{1}{2}} \partial_{t} \boldsymbol{\eta}^{i+\frac{1}{2}}\right\|_{L^{2}(\Omega)} \leq C\left(h^{k}\|\boldsymbol{u}\|_{L^{\infty}\left(H^{k}(\Omega)\right)}+\Delta t^{2}\left\|\frac{\partial^{3} \boldsymbol{u}}{\partial t^{3}}\right\|_{L^{1}\left(0, T ; L^{2}(\Omega)\right)}\right) .
$$

We bound the last term by

$$
\begin{aligned}
\Delta t \sum_{i=0}^{N}\left\|\boldsymbol{R}^{i}\right\|_{L^{2}(\Omega)} \leq & C\|\boldsymbol{R}\|_{l^{\infty}\left(L^{2}\right)} \\
\leq & C \Delta t \sum_{i=1}^{N}\left\|\boldsymbol{r}^{i}\right\|_{L^{2}(\Omega)}+C\left\|\rho\left(\Pi_{h} \boldsymbol{u}_{1}-\boldsymbol{u}_{1}\right)\right\|_{L^{2}(\Omega)} \\
& +C\left\|\frac{1}{2 \Delta t} \int_{0}^{\Delta t} \rho(\Delta t-t)^{2} \frac{\partial^{3} \boldsymbol{u}}{\partial t^{3}}(t) d t\right\|_{L^{2}(\Omega)}
\end{aligned}
$$

We obtain a bound on $\left\|\boldsymbol{r}^{i}\right\|_{L^{2}(\Omega)}$ by first expanding $\boldsymbol{r}^{i}$ as

$$
\boldsymbol{r}^{i}=\rho\left(\boldsymbol{u}_{t t}^{i}-\partial_{t}^{2} \boldsymbol{u}^{i}\right) .
$$

We note that

$$
\partial_{t}^{2} \boldsymbol{u}^{i}=\frac{\boldsymbol{u}^{i+1}-2 \boldsymbol{u}^{i}+\boldsymbol{u}^{i-1}}{\Delta t^{2}}
$$


and use Taylor series to expand $\boldsymbol{u}^{i+1}$ and $\boldsymbol{u}^{i-1}$. We have

$$
\begin{aligned}
\boldsymbol{u}^{i-1}= & \boldsymbol{u}\left(t_{i}-\Delta t\right) \\
= & \boldsymbol{u}\left(t^{i}\right)-\Delta t \frac{\partial \boldsymbol{u}}{\partial t}\left(t^{i}\right)+\frac{\Delta t^{2}}{2} \frac{\partial^{2} \boldsymbol{u}}{\partial t^{2}}\left(t^{i}\right)-\frac{\Delta t^{3}}{6} \frac{\partial^{3} \boldsymbol{u}}{\partial t^{3}}\left(t^{i}\right) \\
& +\frac{1}{6} \int_{t^{i}-\Delta t}^{t^{i}}\left(t^{i}-\Delta t-t\right)^{3} \frac{\partial^{4} \boldsymbol{u}}{\partial t^{4}}(t) d t
\end{aligned}
$$

and

$$
\begin{aligned}
\boldsymbol{u}^{i+1}= & \boldsymbol{u}\left(t_{i}+\Delta t\right) \\
= & \boldsymbol{u}\left(t^{i}\right)+\Delta t \frac{\partial \boldsymbol{u}}{\partial t}\left(t^{i}\right)+\frac{\Delta t^{2}}{2} \frac{\partial^{2} \boldsymbol{u}}{\partial t^{2}}\left(t^{i}\right)+\frac{\Delta t^{3}}{6} \frac{\partial^{3} \boldsymbol{u}}{\partial t^{3}}\left(t^{i}\right) \\
& +\frac{1}{6} \int_{t^{i}}^{t^{i}+\Delta t}\left(t^{i}+\Delta t-t\right)^{3} \frac{\partial^{4} \boldsymbol{u}}{\partial t^{4}}(t) d t
\end{aligned}
$$

so that

$$
\begin{aligned}
\boldsymbol{u}^{i+1}+\boldsymbol{u}^{i-1}=2 \boldsymbol{u}\left(t^{i}\right)+\Delta t^{2} \frac{\partial^{2} \boldsymbol{u}}{\partial t^{2}}\left(t^{i}\right)+\frac{1}{6}[ & {\left[\int_{t^{i}}^{t^{i}+\Delta t} \frac{\partial^{4} \boldsymbol{u}}{\partial t^{4}}(t)\left(t^{i}+\Delta t-t\right)^{3} d t\right.} \\
& \left.+\int_{t^{i}-\Delta t}^{t^{i}} \frac{\partial^{4} \boldsymbol{u}}{\partial t^{4}}(t)\left(t^{i}-\Delta t-t\right)^{2} d t\right] \\
= & 2 \boldsymbol{u}\left(t^{i}\right)+\Delta t^{2} \frac{\partial^{2} \boldsymbol{u}}{\partial t^{2}}\left(t^{i}\right)+\frac{1}{6} \int_{-\Delta t}^{\Delta t}(|t|-\Delta t)^{3} \frac{\partial^{4} \boldsymbol{u}}{\partial t^{4}}\left(t^{i}+t\right) d t .
\end{aligned}
$$

When we use (5.33) and (5.32) in (5.31) we get

$$
\begin{aligned}
\boldsymbol{r}^{i} & =\rho\left(\frac{\partial^{2} \boldsymbol{u}}{\partial t^{2}}\left(t^{i}\right)-\partial_{t}^{2} \boldsymbol{u}^{i}\right) \\
& =\frac{\rho}{6 \Delta t^{2}} \int_{-\Delta t}^{\Delta t}(|t|-\Delta t)^{3} \frac{\partial^{4} \boldsymbol{u}}{\partial t^{4}}\left(t^{i}+t\right) d t
\end{aligned}
$$

and thus

$$
\boldsymbol{r}^{i}=\rho\left(\frac{\partial^{2} \boldsymbol{u}}{\partial t^{2}}\left(t^{i}\right)-\partial_{t}^{2} \boldsymbol{u}^{i}\right)=\frac{\rho}{6 \Delta t^{2}} \int_{-\Delta t}^{\Delta t} \frac{\partial^{4} \boldsymbol{u}}{\partial t^{4}}\left(t^{i}+\Delta t\right)(|t|-\Delta t)^{3} d t .
$$

Therefore

$$
\begin{aligned}
\left\|\boldsymbol{r}^{i}\right\|_{L^{2}(\Omega)}^{2} & \leq C \Delta t^{2} \int_{\Omega}\left[\int_{t^{i}-\Delta t}^{t^{i}+\Delta t} \frac{\partial^{4} \boldsymbol{u}}{\partial t^{4}}(t)\right]^{2} d t \\
& \leq C \Delta t^{3} \int_{t^{i}-\Delta t}^{t^{i}+\Delta t}\left\|\rho^{\frac{1}{2}} \frac{\partial^{4} \boldsymbol{u}}{\partial t^{4}}\right\|_{L^{2}(\Omega)}^{2} \\
& \leq C \Delta t^{4}\left\|\rho^{\frac{1}{2}} \frac{\partial^{4} \boldsymbol{u}}{\partial t^{4}}\right\|_{L^{\infty}\left(L^{2}\right)}^{2},
\end{aligned}
$$

so that

$$
\Delta t \sum_{i=1}^{n}\left\|\boldsymbol{r}^{i}\right\|_{L^{2}(\Omega)} \leq C \Delta t^{2}\left\|\rho^{\frac{1}{2}} \frac{\partial^{4} \boldsymbol{u}}{\partial t^{4}}\right\|_{L^{\infty}\left(L^{2}\right)} \sum_{i=1}^{n} \Delta t \leq C \Delta t^{2}\left\|\rho^{\frac{1}{2}} \frac{\partial^{4} \boldsymbol{u}}{\partial t^{4}}\right\|_{L^{\infty}\left(L^{2}\right)} .
$$


Similarly,

$$
\begin{aligned}
\left\|\frac{1}{2 \Delta t} \int_{0}^{\Delta t} \rho(\Delta t-t)^{2} u^{(3)}(t)\right\|_{L^{2}(\Omega)} & \leq C \Delta t^{2} \int_{\Omega}\left(\int_{0}^{\Delta t} \rho \frac{\partial^{3} \boldsymbol{u}}{\partial t^{3}}(t) d t\right)^{2} \\
& \leq C \Delta t^{3} \int_{0}^{\Delta t}\left\|\rho^{\frac{1}{2}} \frac{\partial^{3} \boldsymbol{u}}{\partial t^{3}}\right\|_{L^{2}(\Omega)}^{2} d t \\
& \leq C \Delta t^{4}\left\|\rho^{\frac{1}{2}} \frac{\partial^{3} \boldsymbol{u}}{\partial t^{3}}\right\|_{L^{\infty}\left(L^{2}\right)}^{2} .
\end{aligned}
$$

Finally, using the approximation result (2.2) and combining all the bounds, we get

$$
\Delta t \sum_{i=0}^{N}\left\|\boldsymbol{R}^{i}\right\|_{L^{2}(\Omega)} \leq C\left(h^{k}+\Delta t^{2}\right),
$$

which concludes the proof of the discrete estimate.

Remark. The convergence rate in space can be at most quadratic, as $\Delta t<C h$.

6. Conclusions. We have developed a priori error estimates for mixed finite element displacement formulations of the acoustic wave equation. Our scheme maintains the same computational complexity as earlier mixed finite element formulations for second order hyperbolic equations, as we have not introduced any additional unknowns. Our formulations require less regularity on the displacement than standard approaches.

We have shown convergence of the scheme via our continuous-in-time estimates, and we have shown that in the temporally discrete case we expect a quadratic convergence rate.

Acknowledgment. The authors would like to thank Dr. Mrinal Sen of the Institute for Geophysics at the University of Texas at Austin for his insights into this problem.

\section{REFERENCES}

[1] R. A. Adams, Sobolev Spaces, Pure and Appl. Math. 65, Academic Press, New York, 1975.

[2] G. A. BAKER, Error estimates for finite element methods for second order hyperbolic equations, SIAM J. Numer. Anal., 13 (1976), pp. 564-576.

[3] H. Bao, J. Bielak, O. Ghattas, L. F. Kallivokas, D. R. O'Hallaron, J. R. Shewchuk, AND J. XU, Large-scale simulation of elastic wave propagation in heterogeneous media on parallel computers, Comput. Methods Appl. Mech. Engrg., 152 (1998), pp. 85-102.

[4] F. Brezzi, J. Douglas, Jr., M. Fortin, and L. Marini, Efficient rectangular mixed finite elements in two and three space variables, RAIRO Modél. Math. Anal. Numér., 21 (1987), pp. 581-604.

[5] F. Brezzi, J. Douglas, Jr., and L. D. Marini, Two families of mixed elements for second order elliptic problems, Numer. Math., 88 (1985), pp. 217-235.

[6] F. Brezzi And M. Fortin, Mixed and Hybrid Finite Element Methods, Springer-Verlag, New York, 1991.

[7] L. C. Cowsar, T. F. Dupont, and M. F. Wheeler, A priori estimates for mixed finite element methods for the wave equation, Comput. Methods App. Mech. Engrg. 82 (1990), pp. 205-222.

[8] L. C. Cowsar, T. F. Dupont, And M. F. Wheeler, A priori estimates for mixed finite element approximations of second-order hyperbolic equations with absorbing boundary conditions, SIAM J. Numer. Anal., 33 (1996), pp. 492-504. 
[9] T. Dupont, $L^{2}$-estimates for Galerkin methods for second order hyperbolic equations, SIAM J. Numer. Anal., 10 (1973), pp. 880-889.

[10] R. W. Graves, Simulating seismic wave propagation in 3D elastic media using staggered-grid finite differences, Bull. Seismol. Soc. Amer., 86 (1996), pp. 1091-1106.

[11] C. Johnson, Discontinuous Galerkin finite element methods for second order hyperbolic problems, Comput. Methods App. Mech. Engrg. 107 (1993), pp. 117-129.

[12] R. J. Knops and L. E. Payne, Uniqueness Theorems in Linear Elasticity, Springer Tracts Nat. Philos. 19, Springer-Verlag, Berlin, 1971.

[13] K. J. MARfurT, Accuracy of finite-difference and finite-element modeling of the scalar and elastic wave equations, Geophysics, 49 (1984), pp. 533-549.

[14] R. McOwen, Partial Differential Equations: Methods and Applications, Prentice-Hall, Upper Saddle River, NJ, 1995.

[15] J. C. Nedelec, Mixed finite elements in $\mathcal{R}^{3}$, Numer. Math., 35 (1980), pp. 315-341.

[16] R. A. Raviart and J. M. Thomas, Mathematical Aspects of the Finite Element Method, Lecture Notes in Math., 106, Springer-Verlag, Berlin, 1997, pp. 292-315.

[17] B. Rivière and M. Wheeler, Discontinuous Finite Element Methods for Acoustic and Elastic Wave Problems. Part I: Semidiscrete Error Estimates, TICAM report 01-02, University of Texas, Austin, TX, 2001.

[18] F. J. Sabadell, F. J. Serón, and J. Badal, A parallel laboratory for simulation and visualization of seismic wavefields, Geophys. Prospecting, 48 (2000), pp. 377-398.

[19] M. K. Sen and P. L. Stoffa, Global Optimization Methods in Geophysical Inversion, Elsevier Science, Amsterdam, The Netherlands, 1995. 